Bulgarian Academy of Sciences. Space Research and Technology Institute.

Aerospace Research in Bulgaria. 32, 2020, Sofia

DOI: https://doi.org/10.3897/arb.v32.e10

\title{
TRANSONIC FLOW STUDY IN A CENTRIFUGAL COMPRESSOR USING PERFORATED AIRFOILS
}

\author{
Bogdan Gherman $^{1}$, Oana Dumitrescu ${ }^{1,2}$, Valeriu Dragan ${ }^{1}$ \\ ${ }^{I}$ National Research and Development Institut for Gas Turbine COMOTI - Romania \\ ${ }^{2}$ University POLITEHNICA of Bucharest \\ e-mail: bogdan.gherman@comoti.ro
}

Key words: Centrifugal Compressor, Numerical Simulation, Airfoil

\begin{abstract}
In this paper, the effect on a perforated plate on a centrifugal compressor is investigated using numerical simulation. The performances and fluid flow are compared for two different vaned diffusers, a base case where there are no perforated plates and a case where a perforated plate is inserted in the rotor casing at exit and the stator blade. The impact of this geometry change on the compressor performances it is studied in the case of adverse angles of attack at stator vane. Near wall refinement ensures the accuracy resolution of the boundary layer with a y+value of one unit corroborated with a growth ratio of 1.1:1. Menter's SST model was used in all numerical cases performed, as implemented in ANSYS CFX. Following the results of the baseline diffuser, the optimal position of the perforated cavity was determined.
\end{abstract}

\section{Introduction}

In recent decades, the compressor design technique has been constantly improved, especially due to the development of CFD technology. Significant improvements in the performance of compressors have been achieved by solving three-dimensional equation for the impeller exit flow. Experimental studies and numerical ones have shown that diffuser have a significant importance on stability limit of a centrifugal compressor, depending on the impeller design and the interface between the impeller and diffuser [1].

Performances of compression system are limited by the occurrence of aerodynamic flow instabilities, which may lead to propulsion system instabilities or, in some extreme case, machine damage. Trebinjac et al. [2] analyzed a transonic centrifugal compressor stage, especially the space between the vaneless and semi-vaneless space where the surge originates. Komatsubara [3] investigate the surge and rotating stall in a centrifugal compressor using a phase portrait reconstruction method, to clarify the cycle behavior of surge. Another study was realized by Bousquet et al [4] who analyzed a 2.5 pressure ratio centrifugal 
compressor stage to achieve a comprehensive description of the flow field from peak efficiency to near stall. In the work by Lennemann and Howard [5], on the appearance of stall, the hydrogen bubble technique is used to determine the flow pattern in an impeller which is in stall. Jansen [6] was probably the first to show that the separation of the boundary layer precedes the initiation of the surge into a vaneless stator. He was able to anticipate cell velocity and speed distribution, but not the number of cells.

Surge into the vaned diffuser is initiated in the vaneless space at the entrance into the diffuser. Due to the increase in static pressure and the increased incidence angle on the leading edge, the flow decreases. Together with the expanded range of vaneless diffusers, the specific speed of the rotor significantly influences the behavior of the stage in unstable conditions at low mass flow rates. A specific low velocity (corresponding to a low flow rate coefficient) can lead to unstable rotor under low flow conditions [7]. This is supported by the fact that the angle of the volute tongue is much more important in the case of high specific speed stages [8]. The conventional approach to pumping industry is to maintain a safety margin on a pump anticipation line (usually $10 \%$ of the air flow rate: which means that the distance from the pump line to the edge is $10 \%$ of the flow rate If the pumping line is at $0.5 \mathrm{~kg} / \mathrm{s}$, then the pumping edge is $0.55 \mathrm{~kg} / \mathrm{s}$ ).

In the cases of high-pressure ratio stages, the speed of sound may be exceeding, thus leading to formation of shock waves. Hence, control of shock waves plays an important role in high speed compressor performance. Savu [9] proved that a perforated airfoil can reduce shock wave intensity and the noise associated with it. Fig. 1 depicts the concept geometry and a shadowgraph proof of concept. Also, porous layers are used to control the incompressible flow around bodies, reducing drag. Bruneau et al. [10] developed a passive control method, in order to establish the proper position of a porous layer, having as limits being the fluid and the bluff-body. A suitable position of porous layer, can lead to a $40 \%$ decreasing in drag coefficient. Another aspect is the complexity of structured grids for porous geometries leading to high computational costs. Thus, Frink [11] developed a new boundary condition, eliminating the grid construction for the plenum, simplifying the model. Solving the Navier-Stockes equations, the influence of porous surfaces on aerodynamic performances was analyzed for supercritical flow [14]. Experimental measuraments revealed that for porous airfoils displacement thickness increases with a lower flow resistance [15].

Studies regarding shock wave control, shape and position of bumps, slots and other passive control devices have been realised over the years, having as purpose a weaker lambda shock wave. Position and bump shape were investigated by Zhou [12] using a sequential quadratic programming optimization method coupled with adjoint method. Another approach regarding porous surfaces is Drăgan's [13], which based on super circulation and Coandă effect, developed a new rotary wing concept, with a channel imbedded inside the wing. 


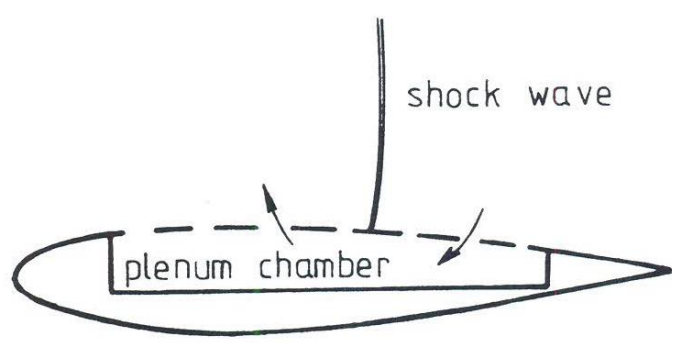

a)

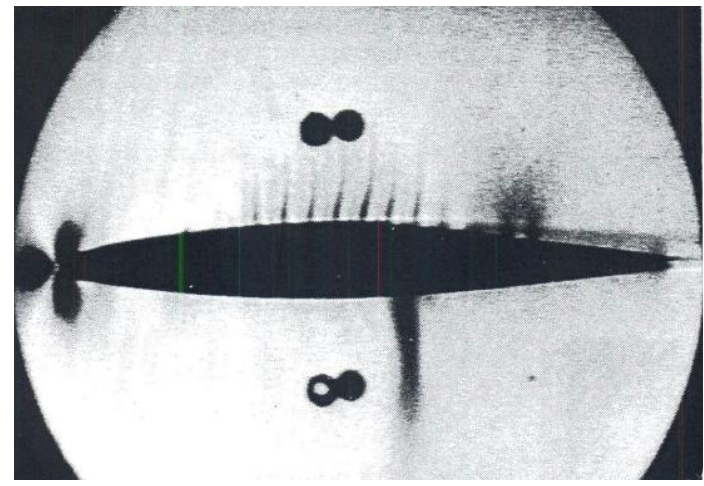

b)

Fig. 1. Suppression of shocks by a permeable surface concept airfoil (a) and Schlieren image of the experiment (b) $(M=0.785, \alpha=00)$ [9]

The main focus of the research is to determine the effectiveness of a perforated cavity incorporated on a rotor casing surface and diffuser vane surface, for transition regime flow mitigation and increase perfomances at adverse angles of attack, see Fig. 3. This comoressor that it is studied here it is subject to extreme conditions near surge. In this case a steady regime it is chosen to quatify as a first step the behavior of porous solution and the mass flow that it is need to modify the flow structure. The idea is to obtain a stable regime with an extra connection between rotor and stator. The air will be extracted from the rotor casing near the exit, where the flow is fully turbulent and secondary flow and tip flow are occurring. It will be studied the possibility to insert the air extracted from rotor casing into the stator blade porous section. In order to provide quantitative data for this type of passive control device, numerical simulations were conducted and compared with a normal diffuser vane. 


\section{Computational modeling}

Compressor design specifications are presented in Table 1, mentioning number of blades, diameters and boundary conditions. For the present paper, just one channel was numerical studied, Fig. 2, in order to validated the concept.

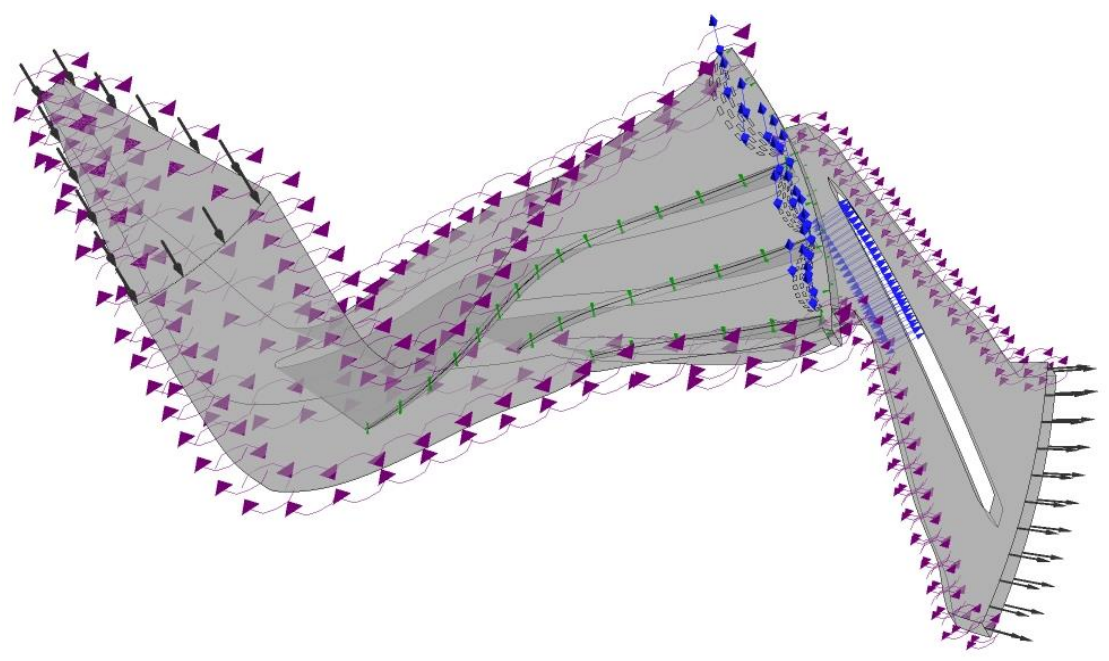

Fig. 2. Computational domain of the centrifugal diffuser

Table 1 presents the specific parameters of the centrifugal compressor.

Table 1. Specifications of the compressor

\begin{tabular}{|l|c|}
\hline \multicolumn{2}{|c|}{ Design operating condition } \\
\hline Inlet pressure & $1 \mathrm{bar}$ \\
\hline Inlet temperature & $288 \mathrm{~K}$ \\
\hline Pressure ratio & 3.5 \\
\hline Design rotational speed & $37550 \mathrm{rpm}$ \\
\hline Design mass flow rate & $0.7 \mathrm{~kg} / \mathrm{s}$ \\
\hline \multicolumn{2}{|c|}{ Impeller } \\
\hline Blade count & $\begin{array}{c}11 \mathrm{main}+11 \mathrm{splitter} \\
+11 \mathrm{splitter}\end{array}$ \\
\hline Inlet diameter & 110.8 \\
\hline Exit diameter & 219 \\
\hline Exit blade height & $6.4 \mathrm{~mm}$ \\
\hline \multicolumn{2}{|c|}{ Vaned diffuser } \\
\hline Blade count & 18 \\
\hline Outlet diameter & $298.8 \mathrm{~mm}$ \\
\hline Vanes height & $5.6 \mathrm{~mm}$ \\
\hline
\end{tabular}


Impeller and diffuser, spatial discretization was performed using a structured multi-block meshing strategy, realized by the mesh generator for turbomachinery CFX TurboGrid. In order to capture the viscous and turbulent effects near the compressor blades and wall, the mesh discretization is performed such as $y+$ is equal to 1 , increasing the mesh near the wall. Table 2 presents the mesh statistics.

Table 2. Mesh statistics

\begin{tabular}{|l|c|}
\hline Number of nodes & $1.7 \mathrm{mil}$. \\
\hline Number of elements & $1.63 \mathrm{mil}$. \\
\hline
\end{tabular}

The computational fluid dynamics solver used in this case is ANSYS CFX. As working fluid, ideal gas was chosen. For the blades and hub/shroud walls a nonslip and adiabatic wall boundary conditions are applied. Between the impeller/diffuser a stage connection was established.

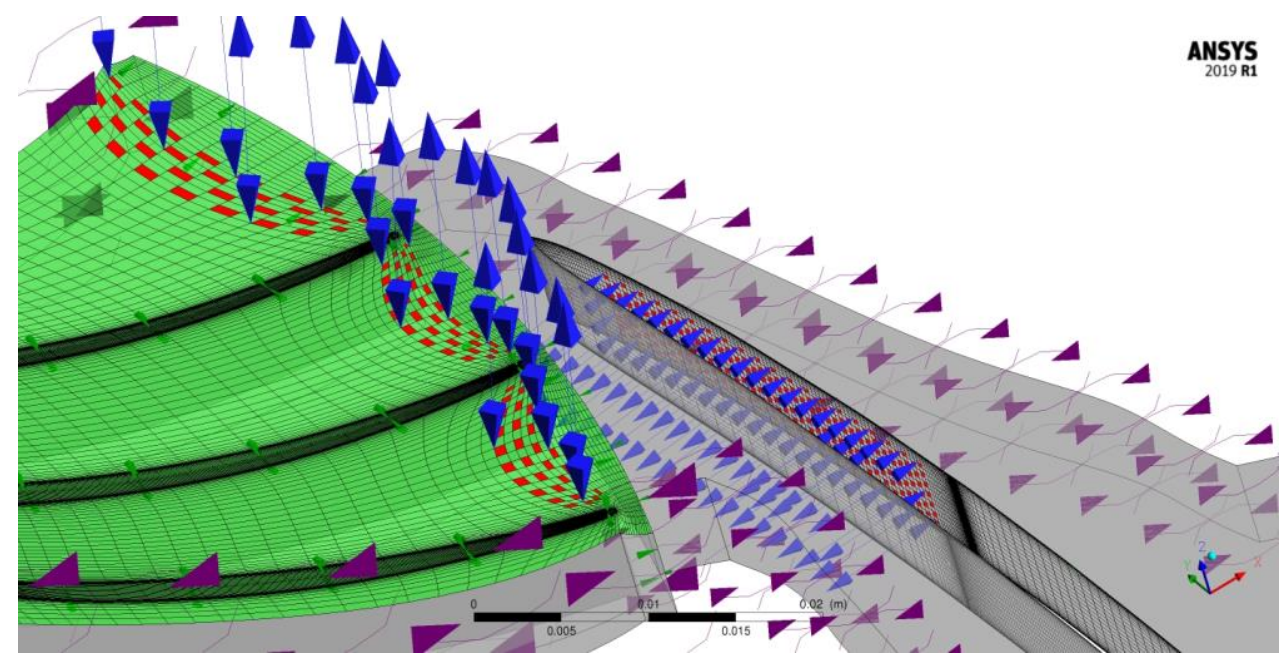

Fig. 3. Grid structure: a) Impeller casing and Diffuser porous zone

The turbulence model used for this analysis is the k-omega SST (Shear Stress Transport). This model has been shown to yield accurate results, and is more suitable for such cases where it is important to capture both the phenomena occurring near the walls and those of the entire field of computing. 
Table 3 illustrates the boundary conditions used for the numerical simulation.

Table 3. Porous Boundary conditions

\begin{tabular}{|l|c|}
\hline \multicolumn{2}{|c|}{ Rotor casing porous boundary condition } \\
\hline Openning & Entrainment \\
\hline Relative Pressure & $1 \mathrm{bar}$ \\
\hline Openning Temperature & $430 \mathrm{~K}$ \\
\hline \multicolumn{2}{|c|}{ Diffuser porous boundary condition } \\
\hline Openning & Entrainment \\
\hline Relative Pressure & $0.8 \mathrm{bar}$ \\
\hline Openning Temperature & $428 \mathrm{~K}$ \\
\hline
\end{tabular}

\section{Results and Discussions}

The numerical results obtain shows a non-transient behavior of the flow when the porous structure it is employed. Also, it was considered that a mass flow of $6 \%$ of the entire mass flow rate should be extracted from the rotor and inserted in the stator blade. The streamlines distribution inside the rotor shows no impact on the flow close to exit in both cases.

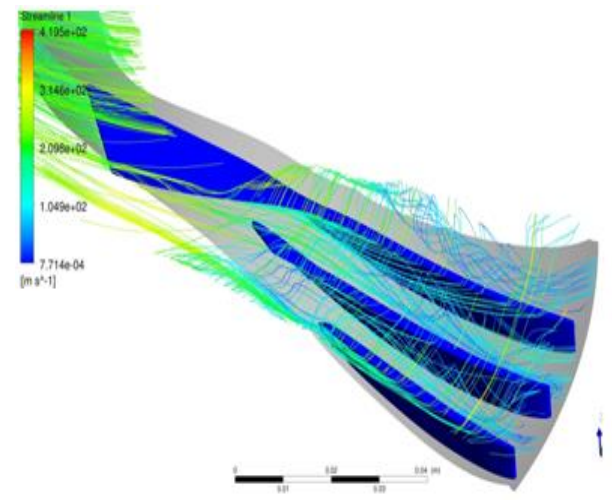

a)

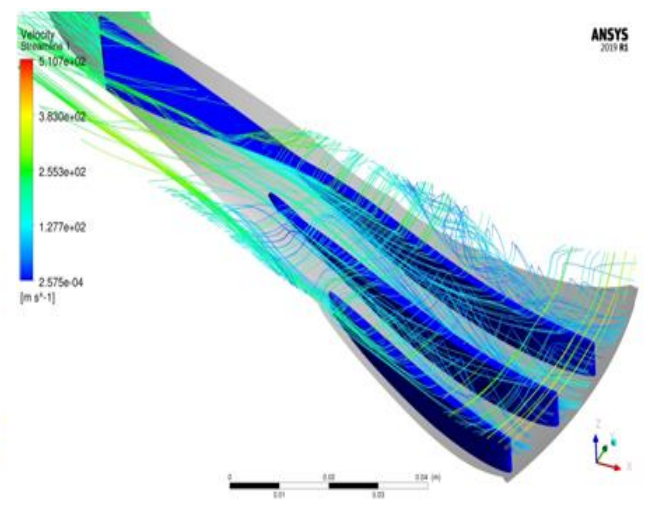

b)

Fig. 4. Streamlines inside rotor: a) with porous, b) base case

Also, the static pressure distribution shows that the porous material has an impact in the region where it is used, but towards the exit of the compressor stage the pressure distribution it is not modified, see Fig. 5. 


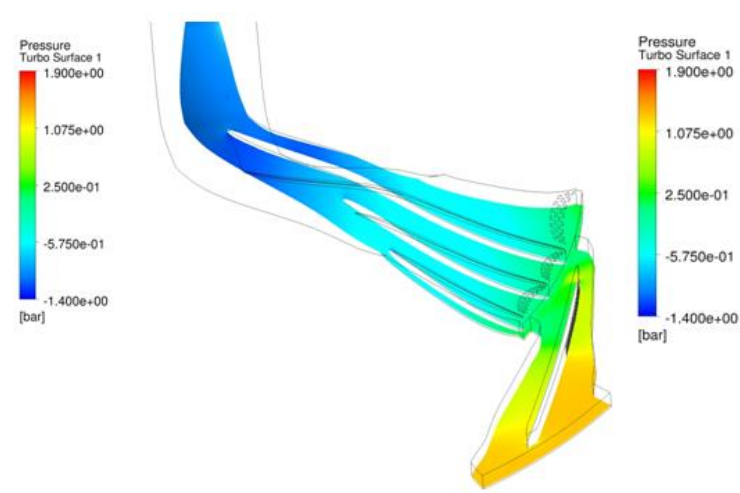

a)

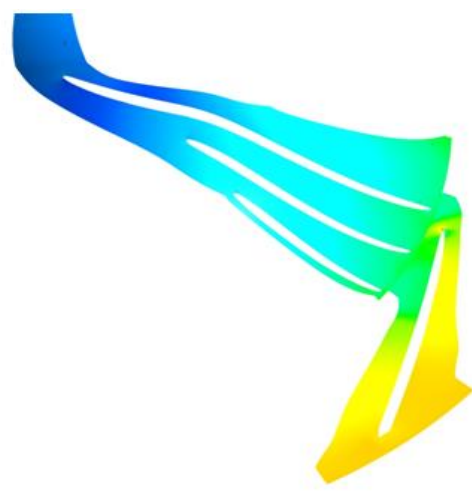

b)

Fig. 5. Pressure distribution inside the domain at 0.5 of the blade height:

a) with porous, b) base case

The compressor regime studied in this case experience an adverse angle of attack at the vane diffuser inlet and a high Mach number; see Fig. 6, 7, and 8. In Fig. 6 it can be seen the low Mach number region on the diffuser blade, suction side. The comparison between the two cases shows that in the porous case (Fig. 6a) the low Mach number region is deacresed and it appears only at the end of the blade.

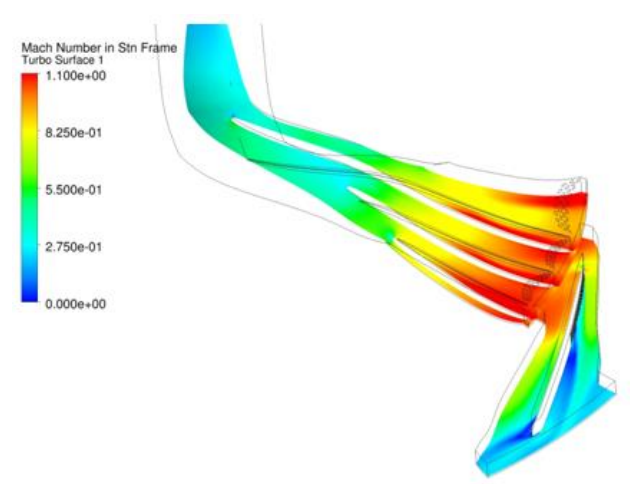

a)

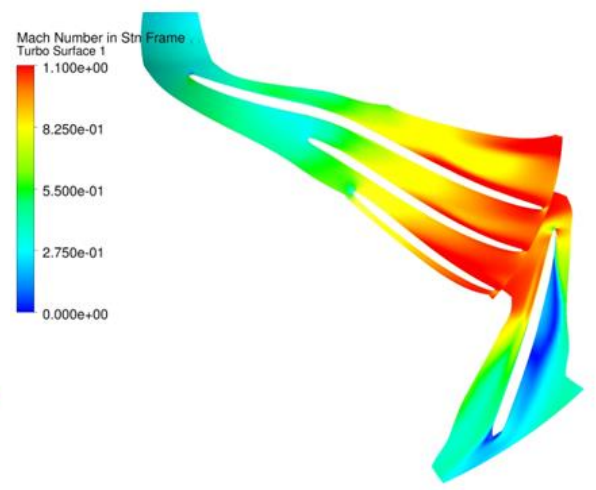

b)

Fig. 6. Mach number distribution at 0.1 of blade height: a) porous case, b) base case

Also, at 0.5 of blade height, Fig. 7, it can be seen that the low Mach region it is still present on the same place. The porous region manages to deacrease the region area, thus increase the mass flow rate through that section. 


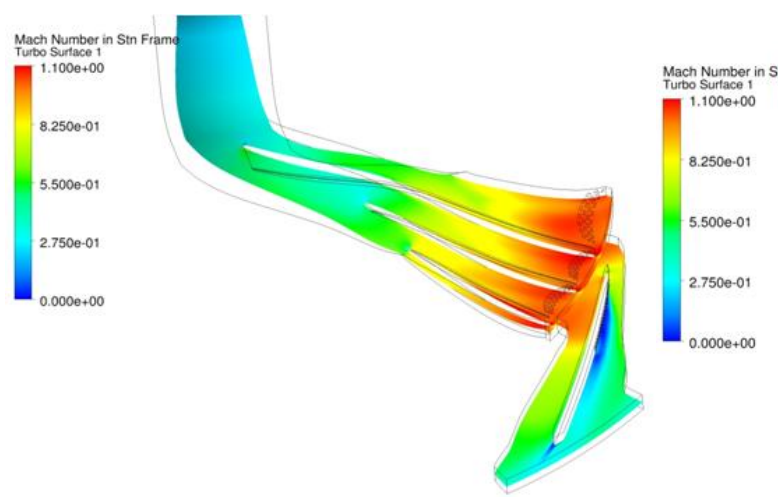

a)

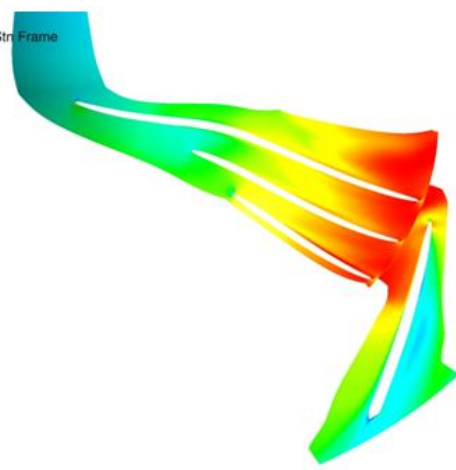

b)

Fig. 7. Mach number distribution at 0.5 of blade height: a) porous case, b) base case

When it comes to the tip of the blade region it can be seen a different scenario, see Fig. 8, when there is no Low Mach number region present in the base case, the extra flow rate that enters through the porous region has a negative impact on the flow structure.

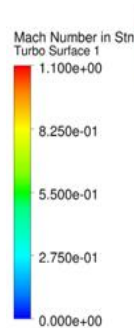

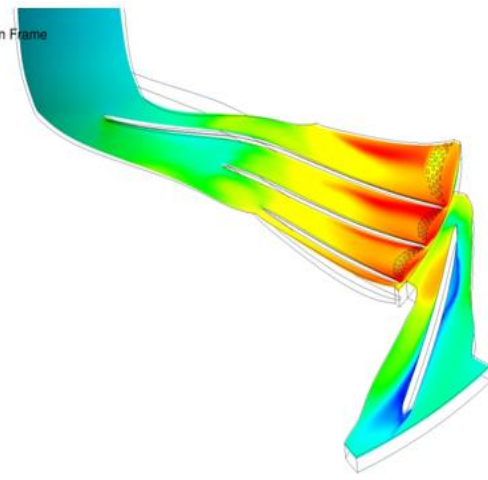

a)

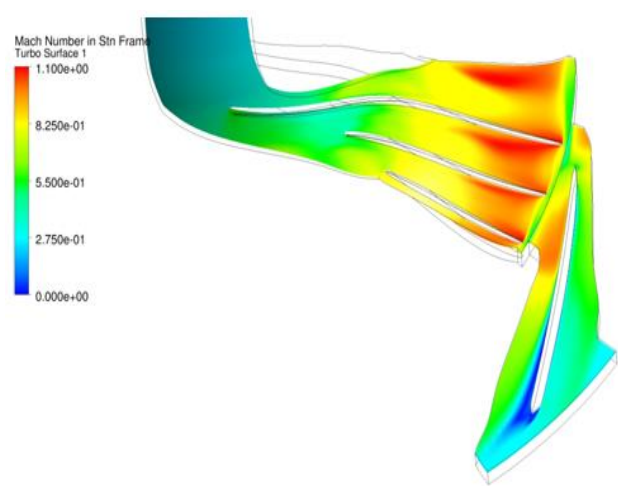

b)

Fig. 8. Mach number distribution at 0.9 of blade height:

a) porous case, b) base case

The idea was to destabilize or reduce the low Mach number zone that forms inside the diffuser by inserting a small amount of air. The results show a mofidication of the low Mach number zone.

Another difference observed between the two cases is the turbulent kinetic energy plot. There it can be observed a high level of this energy where the flow leaves the rotor and enters the vane diffuser. In the porous case there are two 
regions in that are instead of one due to porous material in that region. These two regions have a low level of turbulent kinetic energy compared to the base case.

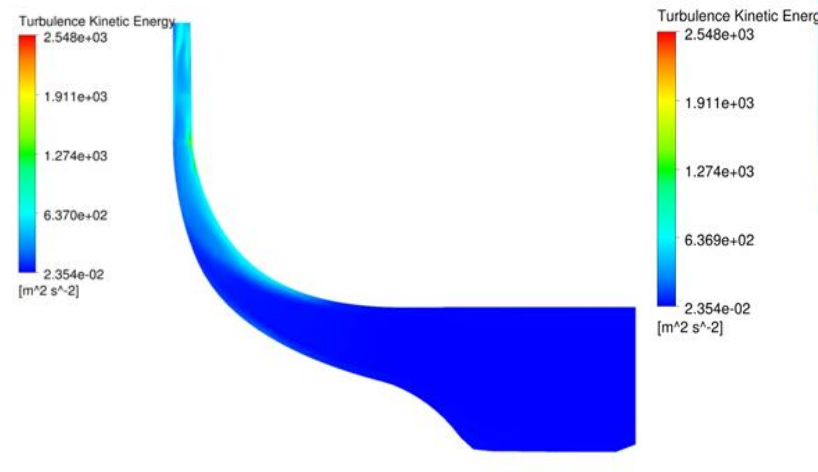

a)

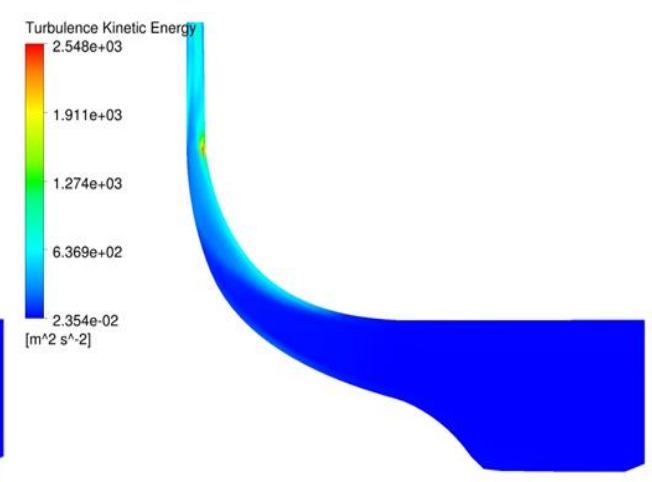

b)

Fig. 9. Turbulent Kinetic Energy distribution on the meridional plane:

a) porous case, b) base case

Also, in Fig. 10 it can be observed a reduction of static entropy levels in the porous case.

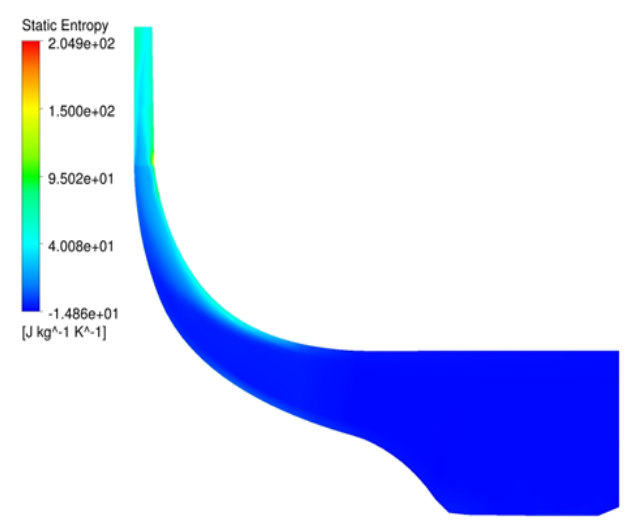

a)

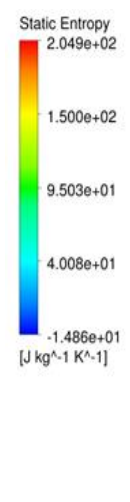

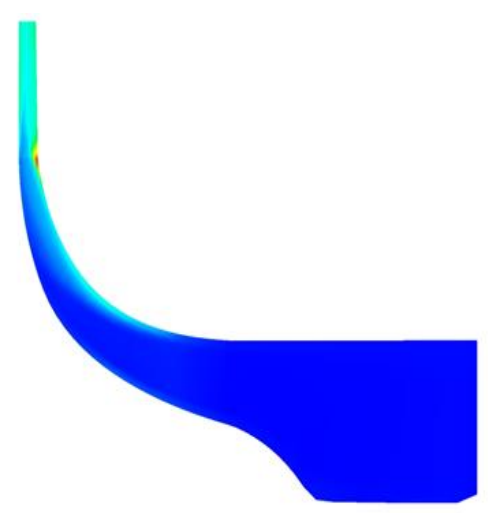

b)

Fig. 10. Static entropy meridional distribution: a) porous case, b) base case

The velocity in stn frame plot shows no difference between the two cases, see Fig. 11. 


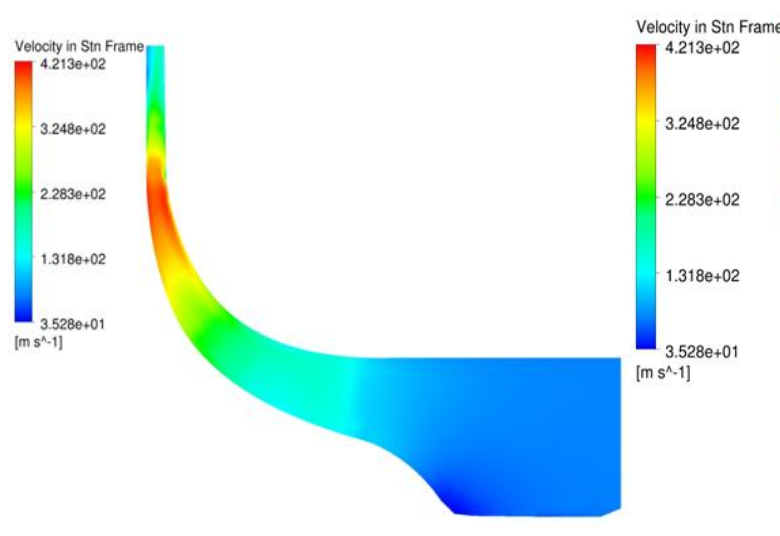

a)

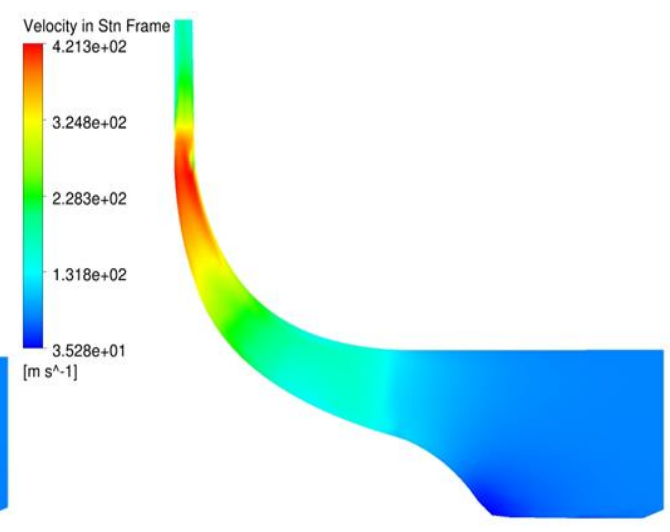

b)

Fig. 11. Velocity in stn frame meridional distribution: a) porous case, b) base case

Another important aspect is the static pressure distribution inside the compressor stage. As it can be seen there is a slite increase at the exit of the rotor in the porous case, however the trend is similar as in the base case, see Fig. 12.

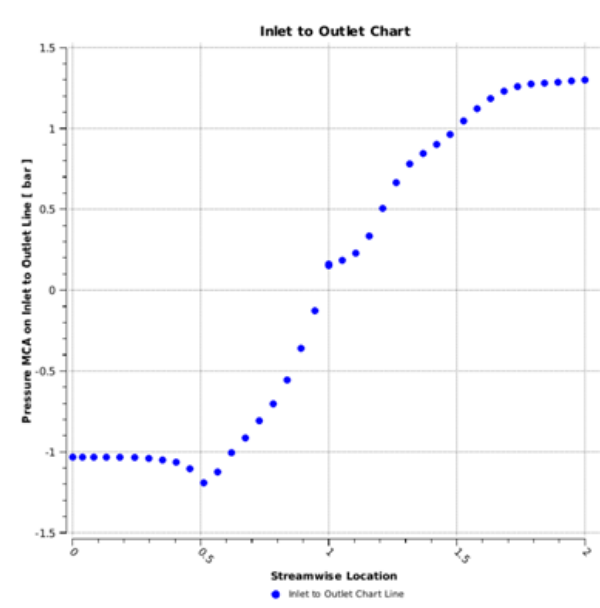

a)

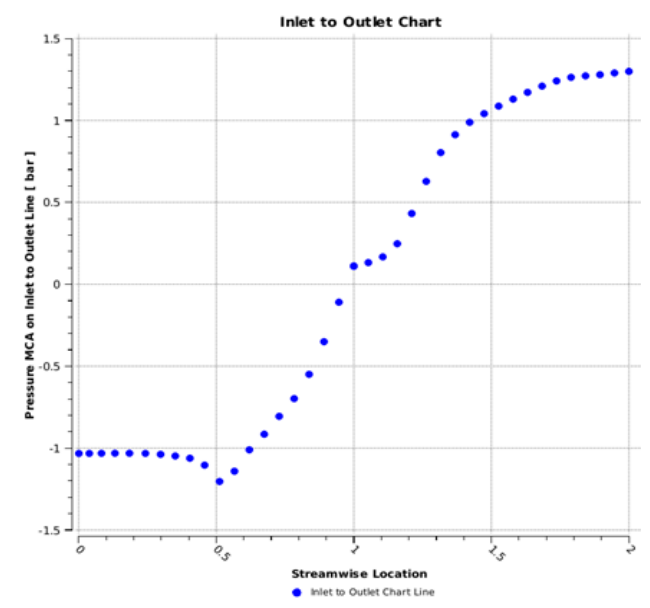

b)

Fig. 12. Static pressure (mass avereged) from inlet to outlet: a) porous case, b) base case

The geometry changes made to the compressor stage modified the performances at this regime, compared to the base case. Although the rotor polytropic efficiency did not change, $\sim 92 \%$, so the extraction of air from the rotor exit had no impact. In the stator case the insertion of air increased the polytropic efficiency $2 \%$, reaching $85 \%$. 


\section{Conclusion}

The numerical analysis performed in this study to see the impact of perforated casing, for rotor, and perforated airfoil in the case of the stator blade. The scenario simulated in this case was to use the air extracted from the rotor exit and inserted in the stator through the perforated airfoil. The idea was to destabilize the low Mach number zone that formed in that area of the blade surface, thus increase the channel witdth. The entrainment boundary condition simulated a perforated airfoil where the pressure was not constant on the entire surface. Thus, where the pressure was higher the mass flow rate entering the stator domain was smaller and viceversa. This can be seen on the entire height of the stator blade. This scenario increased the efficiency on the stator part while did not changed the rotor performances.

In conclusion this idea has potential but more studies have to be performed especially for transient regimes.

\section{References}

1. Waleed, Al-Busaidi, Pericles Pilidis, Techno-Economic Optimization of Diffuser Configuration Effect on Centrifugal Compressor Performance, American Journal of Energy Research, 2015, 3, 2, 37-48.

2. Trebinjac, I., N. Bulot, X. Ottavy, and N. Buffaz, Surge inception' in a transonic centrifugal compressor stage, Proceedings of the ASME Turbo Expo, pp. GT2011-G45116, June 2011.

3. Komatsubara, Y., S. Mizuki, Dynamical System Analysis of Unsteady Phenomena in Centrifugal Compressor, Journal of Thermal Science, 1997, 6, 1.

4. Bousquet, Y., X. Carbonneau, G. Dufour, N. Binder, and I. Trebinjac, Analysis of the Unsteady Flow Field in a Centrifugal Compressor from Peak Efficiency to Near Stall with Full-Annulus Simulations, International Journal of Rotating Machinery, Volume 2014, Article ID 729629, 11. DOI:10.1155/2014/729629

5. McDonald, G. B., E. Lennemann, J. H. G. Howard, Measured and Predicted Flow Near the Exit of a Radial-Flow Impeller, J. Eng. Power, 1971, 93, 4, 441-46. DOI:10.1115/1.3445604

6. Jansen, W., Rotating Stall in a Radial Vaneless Diffuser, J. Basic Eng., 1964, 86, 4, 750-58. DOI:10.1115/1.3655945

7. http://pt.slideshare.net/soulstalker/easa-part-66-module-154compressors?smtNoRedir=1

8. Frigne, P., and R. van den Braembussche, One Dimensional Design of Centrifugal Compressors Taking into Account Flow Separation in the Impeller, Von Karman Institute for Fluid Dynamics Turbomachinery Department, June 1978.

9. Savu, G., Trifu O., and Dumitrescu, L. Z. Supresssion of shocks on transonic airfoils, In: Proceedings of $14^{\text {th }}$ International Symp. "Shock tubes \& Waves", Sydney, Australia, 1983, NSW university press, 1984, 92-101.

10. Bruneau, C. H., I. Mortazavi, Numerical modelling and passive flow control using porous media, Computers \& Fluids, 2007, 37, 5,

DOI:10.1016/j.compfluid.2007.07.001 
11. Frink, N. T., D. L. Bonhaus, V. N. Vatsa, and S. X. S. Bauer, A boundary condition for simulation of flow over porous surfaces, $19^{\text {th }}$ Applied Aerodynamics Conference June 11-14, 2001/Anaheim, California.

12. Ling Zhou, Dehua Chen, Yang Tao, Guangyuan Liu, Shuheng Song, Shidong Zhong, Paasive shock wave/boundary layer control of wing at transonic speeds, Theoretical\&Applied Mechanics Letters, 2017, 7, 325-30.

13. Dragan, V., Contributions Regarding the Design of a Self Super Circulated Rotary Wing, $16^{\text {th }}$ International Conference on Modern Technologies, Quality and Innovation.

14. Jixiang, S., Ling, Z., Xin, P., \& Shidong, Z. Computational Analysis on Porosity Effect on Aerodynamic Characteristic of Supercritical Airfoil, In: $5^{\text {th }}$ International Conference on Intelligent Systems Design and Engineering Applications, 2014, 310-13.

15. Geyer, T., E. Sarradj, and C. Fritzsche, Porous Airfoils: Noise Reduction and Boundary Layer Effects, $15^{\text {th }}$ AIAA/CEAS Aeroacoustics Conference (30 ${ }^{\text {th }}$ AIAA Aeroacoustics Conference), 11-13 May 2009, Miami, Florida. 